PROCEEDINGS OF THE

AMERICAN MATHEMATICAL SOCIETY

Volume 132, Number 5, Pages 1385-1388

S 0002-9939(03)07095-3

Article electronically published on December 5, 2003

\title{
THE LINEAR ESCAPE LIMIT SET
}

\author{
CHRISTOPHER J. BISHOP
}

(Communicated by Juha M. Heinonen)

\begin{abstract}
If $G$ is any Kleinian group, we show that the dimension of the limit set $\Lambda$ is always equal to either the dimension of the bounded geodesics or the dimension of the geodesics that escape to infinity at linear speed.
\end{abstract}

Suppose $G$ is a discrete group of isometries on hyperbolic space $\mathbb{B}^{n}, n \geq 2$. The limit set $\Lambda \subset S^{n-1}$ is defined to be the accumulation set of the $G$-orbit of $0 \in \mathbb{B}^{n}$. A point $x \in \Lambda$ can be associated to the radial segment that ends at $x$, which in turn projects to a geodesic ray $\gamma$ (based at $z_{0}$, the projection of 0 ) in the quotient $M=\mathbb{B}^{n} / G$. We then write $\Lambda$ as the disjoint union $\Lambda_{c} \cup \Lambda_{e}$, where $\Lambda_{c}$ (the "conical limit set") corresponds to $\gamma$ 's that return to some compact set at arbitrarily large times and $\Lambda_{e}$ (the "escaping limit set") corresponds to $\gamma$ 's that eventually leave every compact set. Obviously,

$$
\operatorname{dim}(\Lambda)=\max \left(\operatorname{dim}\left(\Lambda_{c}\right), \operatorname{dim}\left(\Lambda_{e}\right)\right)
$$

(where dim denotes Hausdorff dimension). The purpose of this note is to show that this equality is still true if we replace both $\Lambda_{c}$ and $\Lambda_{e}$ by certain subsets.

Let $\Lambda_{b}$ (the "bounded limit set") be the subset of $\Lambda_{c}$ corresponding to $\gamma$ 's that remain bounded for all time. Parametrize geodesic rays by hyperbolic arclength and for $0<\alpha<1$, let $\Lambda_{\alpha}$ correspond to geodesic rays $\gamma$ such that

$$
\liminf _{t} \frac{\operatorname{dist}_{M}\left(\gamma(t), z_{0}\right)}{t}>\alpha
$$

and let $\Lambda_{\ell}=\bigcup_{0<\alpha<1} \Lambda_{\alpha}$ denote the "linear escape limit set". Related sets have been considered by Lundh in [2] $\left(\Lambda \backslash \Lambda_{\alpha}=\mathcal{L}\left(\frac{1}{1+\alpha}\right)\right.$ where $\mathcal{L}$ is as in Definition 3.14 of [2]).

Theorem 1. For any discrete group $G, \operatorname{dim}(\Lambda)=\max \left(\operatorname{dim}\left(\Lambda_{b}\right), \operatorname{dim}\left(\Lambda_{\ell}\right)\right)$.

In other words, the dimension of $\Lambda$ is determined either by the geodesic rays that stay bounded for all time or by those that escape to $\infty$ at the fastest possible speed. This is somewhat surprising since neither of these behaviors is "typical" in general. For example, if $n=2$ and $M$ is a finite area Riemann surface that is not compact, then $\Lambda_{c}$ will have full Lebesgue measure but $\Lambda_{b}$ will have measure zero (e.g., see [5]).

Received by the editors May 22, 2002 and, in revised form, October 30, 2002.

2000 Mathematics Subject Classification. Primary 30F35.

Key words and phrases. Hausdorff dimension, quasi-Fuchsian groups, quasiconformal deformation, critical exponent, convex core.

The author was partially supported by NSF Grant DMS 0103626. 
If $G$ is elementary, then $\operatorname{dim}(\Lambda)=0$ and there is nothing to do. The Poincaré exponent $\delta$ of $G$ is defined as a critical exponent of convergence of the Poincaré series, i.e.,

$$
\delta=\inf \left\{s: \sum_{g \in G} e^{-s \rho(0, g(0))}<\infty\right\}
$$

and in [1] it is shown that for non-elementary $G$, we have $\delta=\operatorname{dim}\left(\Lambda_{c}\right)=\operatorname{dim}\left(\Lambda_{b}\right)$. Theorem 1 follows from the following result (which is similar to Theorem 2.1.1 of [5]).

Lemma 2. Suppose $G$ is a discrete group of isometries on $\mathbb{B}^{n}, n \geq 2$ and $0<\alpha<1$ and assume that $\Lambda \backslash \Lambda_{\alpha}$ supports a positive measure $\mu$ such that

$$
\mu(B(x, r)) \leq \varphi(r)
$$

for all balls and some increasing function $\varphi$ that satisfies $\varphi(A t) \leq B \varphi(t)$ for some $A>1, B<\infty$. Then

$$
\sum_{g \in G} \varphi\left((1-|g(0)|)^{(1+\beta)^{-1}}\right)=\infty
$$

for every $\beta>\alpha$.

We will prove this later. First we deduce a few consequences.

Corollary 3. If $G$ is a discrete group of isometries on $\mathbb{B}^{n}, n \geq 2$ and $0<\alpha<1$, then $\delta \geq \operatorname{dim}\left(\Lambda \backslash \Lambda_{\alpha}\right) /(1+\alpha)$.

Proof. If $s=\operatorname{dim}\left(\Lambda \backslash \Lambda_{\alpha}\right)$, then Frostman's lemma (e.g., 3]) says it supports a measure satisfying (11) with $\varphi(t)=t^{s-\epsilon}$ for every $\epsilon>0$. By (2), $\delta \geq(s-\epsilon) /(1+\beta)$. Taking $\epsilon \rightarrow 0$ gives the result.

Corollary 4. For any non-elementary group $G$ we have $\delta=\operatorname{dim}\left(\Lambda \backslash \Lambda_{\ell}\right)$.

Proof. Take $\alpha \rightarrow 0$ in the previous result to get " $\geq$ ". Since $\delta=\operatorname{dim}\left(\Lambda_{c}\right)$ and $\Lambda_{c} \subset \Lambda \backslash \Lambda_{\ell}$, the other direction is clear.

Proof of Theorem 1. Our previous remarks imply that

$\operatorname{dim}(\Lambda)=\max \left(\operatorname{dim}\left(\Lambda \backslash \Lambda_{\ell}\right), \operatorname{dim}\left(\Lambda_{\ell}\right)\right)=\max \left(\delta, \operatorname{dim}\left(\Lambda_{\ell}\right)\right)=\max \left(\operatorname{dim}\left(\Lambda_{b}\right), \operatorname{dim}\left(\Lambda_{\ell}\right)\right)$.

Let $|E|_{n}$ denote the $n$-dimensional Lebesgue measure of the set $E$.

Corollary 5. If $G$ is a discrete group of isometries on $\mathbb{B}^{n+1}$ and $|\Lambda|_{n}>0$, then $\left|\Lambda_{\alpha}\right|_{n}=|\Lambda|_{n}>0$ for every $\alpha<\frac{n}{\delta}-1$.

Proof. Suppose $\alpha$ is such that $\left|\Lambda \backslash \Lambda_{\alpha}\right|_{n}>0$. If we let $\mu$ be $n$-dimensional measure restricted to $\Lambda_{\alpha}$, then it satisfies (1) with $\varphi(t)=t^{n}$; so by Lemma 2, the Poincaré series diverges at $n /(1+\beta)$ for all $\beta>\alpha$. Thus $\delta \geq n /(1+\alpha)$ and hence $\alpha \geq \frac{n}{\delta}-1$, as desired.

A Kleinian group $(n=3)$ is called analytically finite if $\left(S^{2} \backslash \Lambda\right) / G$ is a finite union of finite area Riemann surfaces. By the Ahlfors finiteness theorem, all finitely generated Kleinian groups have this property.

Corollary 6. If $G$ is an analytically finite Kleinian group such that $\operatorname{dim}\left(\Lambda_{b}\right) \neq$ $\operatorname{dim}(\Lambda)$, then $\left|\Lambda_{\alpha}\right|_{2}>0$ for all $\alpha<\frac{2}{\delta}-1$. 
Proof. Corollary 1.4 of [1] implies that such a group has a positive area limit set. So this is a special case of the previous result.

The Ahlfors conjecture claims there are no finitely generated Kleinian groups with $\Lambda \neq S^{2}$ and $|\Lambda|_{2}>0$. However, there are analytically finite examples.

Points of $\Lambda_{\ell}$ are closely related to McMullen's "deep points" (4 4 ). The convex hull of $M=\mathbb{B}^{n} / G$ is the quotient of the hyperbolic convex hull of $\Lambda$ in $\mathbb{B}^{n}$. A point of $\Lambda$ is called a deep point if the corresponding geodesic is such that $\operatorname{dist}(\gamma(t), \partial C(M))$ increases with an eventually linear lower bound. Clearly, all such points are in $\Lambda_{\ell}$ and the two sets coincide if $\partial C(M)$ is compact. In general, however, there can be points of $\Lambda_{\ell}$ that are not deep points (and the deep points can be empty even if $\Lambda_{\ell}$ is not, e.g., in some quasi-Fuchsian groups).

If $\partial C(M)$ is compact and $\left|\Lambda_{\alpha}\right|_{2}>0$, then $\Lambda$ is a bit "thicker" than a general positive area set must be. In particular, if $w \in \Lambda_{\alpha}$, then the largest omitted disk in $\Lambda \cap A_{n}, A_{n}=\left\{w: 2^{-n} \leq|z-w| \leq 2^{-n+1}\right\}$, has diameter $\simeq 2^{-n} \cdot 2^{-\alpha n}$. On the other hand, Cantor sets of positive area can be easily constructed where the largest omitted ball is $\simeq 2^{-n} a_{n}$ for any series such that $\sum_{n} a_{n}<\infty$.

Proof of Lemma国. Let $X_{\alpha}=\Lambda \backslash \Lambda_{\alpha}$. For any $1>\beta>\alpha$, and $x \in X_{\alpha}$, the corresponding geodesic ray satisfies

$$
\operatorname{dist}\left(\gamma\left(t_{n}\right), z_{0}\right) \leq \beta t_{n},
$$

for some sequence $t_{n} \nearrow \infty$ (the sequence may depend on the point $x$ ). Given a disk $D(x, r)$ on $S^{n-1}$, let $z_{D}$ be the point on the radius from 0 to $x$ at (Euclidean) distance $r$ from $S^{n-1}$. By definition, every point of $X_{\alpha}$ is covered by arbitrarily small disks $D$ so that $z_{D}$ satisfies

$$
\rho\left(z_{D}, G(0)\right) \leq \beta \rho\left(z_{D}, 0\right) .
$$

By the Vitali covering theorem, there is a disjoint subcollection of these disks which cover $\mu$-almost every point of $X_{\alpha}$. Let $\left\{D_{n}\right\}$ be an enumeration of this collection, $\left\{z_{n}\right\}$ the corresponding points, and choose $w_{n} \in G(0)$ so that $\rho\left(z_{n}, w_{n}\right) \leq \beta \rho\left(z_{D}, 0\right)$. Let $\mathcal{W}=\bigcup_{n}\left\{w_{n}\right\}$, i.e., is the collection of orbit points chosen.

To proceed further we need a simple lemma about hyperbolic geometry.

Lemma 7. There is $M<\infty$ so that if $z, w \in \mathbb{B}^{n}$ and $\rho(z, w) \leq \beta \rho(0, z)$, then

$$
|z-w| \leq M(1-|w|)^{1 /(1+\beta)}
$$

and

$$
1-|z| \leq M(1-|w|)^{1 /(1+\beta)} .
$$

Proof. Since $\rho(0, w) \leq \rho(0, z)+\rho(z, w) \leq(1+\beta) \rho(0, z)$, we have $\rho(0, z) \geq d \equiv$ $\rho(0, w) /(1+\beta)$. This implies the second estimate since a point in the ball which is a hyperbolic distance $d$ from 0 has Euclidean distance to the boundary $\simeq e^{-d} \simeq$ $(1-|w|)^{1 /(1+\beta)}$.

Let $t=\rho(0, w)-d=\beta d$ and $k=\rho(0, z)-d \geq 0$. If $|z-w|=M(1-|w|)^{1 /(1+\beta)}$, with $M \gg 1$, then the part of the geodesic between $z$ and $w$ that lies inside $\{x: \rho(0, x) \leq d\}$ has hyperbolic length $\geq \log M-C_{2}$ for some absolute $C_{2}$. Thus

$$
\rho(z, w) \geq \log M-C_{2}+k+t=\log M+k+\beta d-C_{2} .
$$

Since we also have $\rho(z, w) \leq \beta \rho(0, z)=\beta(d+k)$, we deduce that $\log M \leq C_{2}+$ $(\beta-1) k \leq C_{2}$ (recall $\beta \leq 1$ ), and the lemma is proven. 
We now continue with the proof of Lemma 2 For each $w \in \mathcal{W}$, let $r_{w}=$ $M(1-|w|)^{1 /(1+\beta)}$, with $M$ as in Lemma 7 . Thus $D_{n} \subset D\left(w^{*}, 4 r_{w}\right)$ and

$$
\sum_{D_{n} \in \mathcal{C}(w)} \mu\left(D_{n}\right) \leq \mu\left(D\left(w^{*}, 2 r_{w}\right)\right) \leq C \varphi\left(r_{w}\right) \leq C \varphi\left((1-|w|)^{\frac{1}{1+\beta}}\right),
$$

where $\mathcal{C}(w)$ is the set of all disks in $\left\{D_{n}\right\}$ associated to the point $w \in \mathcal{W}$. Since the disks $\left\{D_{n}\right\}$ cover full $\mu$ measure,

$$
\|\mu\| \leq C \sum_{w \in \mathcal{W}} \varphi\left((1-|w|)^{\frac{1}{1+\beta}}\right) .
$$

Thus there is a finite subcollection $\mathcal{W}_{1}$ over which the sum is $\geq \frac{1}{2}\|\mu\| / C$. Repeating the argument starting with a covering of $X_{\alpha}$ by disks not in $\mathcal{W}_{1}$ we get a second, distinct, collection $\mathcal{W}_{2}$ with the same property. Continuing by induction we obtain an infinite family of such collections and this obviously implies that (2) diverges.

I thank Torbjörn Lundh and the referee for carefully reading the paper and for many suggestions which greatly improved it.

\section{REFERENCES}

[1] C. J. Bishop and P. W. Jones. Hausdorff dimension and Kleinian groups. Acta. Math. 179:1-39, 1997. MR 98k:22043

[2] T. Lundh. Geodesics on quotient manifolds and their corresponding limit points. Michigan Math. J. 51:279-304, 2003.

[3] P. Mattila. Geometry of sets and measures in Euclidean spaces. Fractals and rectifiability. Cambridge Studies in Advanced Mathematics 44. Cambridge University Press, Cambridge, 1995. MR 96h:28006

[4] C. T. McMullen. Renormalization and 3-manifolds which fiber over the circle. Annals of Mathematics Studies 142. Princeton University Press, Princeton, NJ, 1996. MR 97f:57022

[5] P. J. Nicholls. The ergodic theory of discrete groups. London Mathematical Society Lecture Note Series 143. Cambridge University Press, 1989. MR 91i:58104

Mathematics Department, SUNy at Stony Brook, Stony Brook, New York 11794-3651

E-mail address: bishop@math.sunysb.edu 